Ann. Biol. anim. Bioch. Biophys., r973, 13 (2), 203-2I4.

\title{
ACTION DE LA FLORE BACTÉRIENNE SUR LA MORPHOLOGIE ET LA SURFACE DE LA MUQUEUSE DE L'INTESTIN GRELE DU RAT
}

\author{
J. C. MESLIN, E. SACQUET* et J.-L. GUENET** \\ Station de Recherches de Nutrition, \\ Centre national de Recherches zootechniques, I. N. R. A., \\ 78350 Jouy en Josas \\ * Service des Animaux sans Germes, \\ C. N.R.S., \\ 91190 Gif sur Yvette \\ **Institut de Biologie moléculaire, \\ Institut Pasteur, \\ 75 Paris $15 \mathrm{e}$
}

\section{RÉSUMḾ́}

L'étude comparée de la morphologie de l'intestin grêle, chez le rat axénique d'une part, chez le rat holoxénique d'autre part, fait apparaître d'importantes différences morphologiques qui traduisent l'action de la flore microbienne sur cet organe.

Les différents caractères morphologiques : longueur de la circonférence de l'intestin grêle, surface de la muqueuse, nombre et hauteur des villosités ne sont pas modifiés d'une manière uniforme à tous les niveaux. Chez le sujet axénique la longueur de la circonférence est diminuée dans la région antérieure, inchangée dans la région moyenne, accrue dans la région postérieure, alors que la surface de la muqueuse est semblable dans la région antérieure et diminuée dans les régions moyenne et terminale.

Le nombre des villosités est diminué dans la région antérieure, il est inchangé dans les autres régions, alors que la hauteur des villosités tend à être plus grande dans la région antérieure et plus petite dans les autres régions.

La surface totale de la muqueuse intestinale, exprimée en fonction du poids corporel du rat n'est pas significativement réduite chez le sujet axénique.

La surface de la muqueuse de l'intestin grêle joue un tôle important dans les phénomènes d'absorption. Cette surface peut être modifiée en fonction de l'âge (PERMEZEL et WEBLING, I97I) ou de conditions physiologiques telles que la lactation (BOYNE, FELI, et ROBB, I966). Elle peut être aussi modifiée par la présence ou l'absence de flore microbienne. 
D'après GoRdon (I960, I96I, I966, I968), la muqueuse de l'intestin grêle du rat axénique ( $\left.{ }^{1}\right)$ est caractérisée par des villosités grêles qui possèdent un stroma peu développé. La paroi de l'intestin grêle est moins riche en tissu conjonctif et en eau que celle du sujet holoxénique; sa vascularisation et son irrigation sanguines sont diminuées. Ėnfin, la surface de la muqueuse intestinale serait sensiblement inférieure chez le sujet axénique.

Cependant aucune étude n'a été consacrée à ce sujet depuis les travaux originatux de Gordon et BruckNer-Kardoss (I96I).

Comme l'acquisition de ces données dépend pour une part considérable de la valeur de la technique mise en œuvre, il nous est apparu utile de procéder à une nouvelle étude de la détermination de la surface muqueuse intestinale du rat axénique comparée à celle du rat holoxénique, à l'aide de techniques améliorées.

\section{MATÉRIEL E'T MÉTHODES}

Pour déterminer la surface de la muqueuse intestinale, il est nécessaire de connaître la longueur de l'intestin grêle, sa circonférence, la surface muqueuse moyenne par unité de surface séreuse $(\mathrm{R} a)$, la surface muqueuse moyenne par centimètre de longueur d'intestin $(\mathrm{M} a)$, ces différentes valeurs entrent dans l'équation proposée par Fisher et Parsons (1950). Outre ces valeurs indispensables pour connaître la surface de la muqueuse de l'intestin grêle, nous nous sommes proposé de déterminer le nombre de villosités par centimètre carré de séreuse et par centimètre de longueur d'intestin grêle, ainsi que la hauteur des villosités.

Toutes les valeurs mesurées sont représentées par leur moyenne arithmétique et l'écart-type de cette moyenne. La comparaison de ces moyennes a été faite selon le test de Student.

Les observations sont faites à 3 niveaux de l'intestin grêle :

- Le niveau I est situé à ro p. Ioo de la longueur totale à partir du pylore, ce qui correspond au duodenum distal, le pancréas étant présent sur le prélèvement (chez le rat, les glandes de Brünner ne sont présentes que sur une partie extrêmement réduite du duodénum proximal).

- Le niveau 2 est situé à $50 \mathrm{p}$. Ioo de la longueur totale à partir du pylore et correspond au milieu du jéjunum.

- Le niveau 3 est à $90 \mathrm{p}$. Ioo de la longueur totale à partir du pylore et correspond à la partie terminale de l'iléon.

Les valeurs ainsi mesurées aux niveaux I, 2 et 3 sont utilisées pour calculer les surfaces des premier, deuxième et troisième tiers de l'intestin grêle. La surface totale de l'intestin grêle est estimée en additionnant les valeurs ainsi calculées pour chacun de ces segments intestinaux.

Les détails de la technique se présentent de la façon suivante :

- les sujets d'expérience sont des rats Fischer, mâles, 5 axéniques et 5 holoxéniques, recevant le même régime alimentaire commercial stérilisé, de poids compris entre 190 et $219 \mathrm{~g}$ et de même âge (7o jours).

Les principales étapes du prélèvement de l'intestin grêle sont comme suit :

- anesthésie des animaux à l'éther; ouverture de la paroi abdominale et mise en place d'une canule au niveau du pylore;

- section de l'intestin grêle au niveau de la jonction iléo-cæcale ;

- lavage de la lumière intestinale au sérum physiologique tiède, sous une faible pression, ne dépassant pas $30 \mathrm{~cm}$ d'eau ;

- résection du mésentère sans léser la séreuse, prélèvement de l'intestin grêle et mesure de sa longueur.

(1) Nous utilisons la terminologie proposée par RAIBAUd et al. (I966). 
La fixation des tissus se fait en remplaçant le sérum physiologique par le fixateur toujours sous pression constante, après quoi l'intestin est ligaturé à ses deux extrêmités et immergé dans un flacon rempli de fixateur pendant une période d'au moins I2 heures. Cette fixation entraîne une rétraction; celle-ci est estimée par un coefficient : $r=\frac{\mathrm{L}_{i}-\mathrm{L}_{f}}{\mathrm{~L}_{i}} \times$ roo où $\mathrm{L}_{i}=$ Iongueur initiale, $\mathrm{L}_{f}=$ longueur après fixation. Une étude préliminaire a montré que le fixateur de Bouin entraînait une très forte rétraction de l'ordre de $10,8 \mathrm{p}$. roo. Nous utilisons le fixateur de Serra : alcool éthylique absolu, 6 volumes ; formol du commerce, 3 volumes ; acide acétique cristallisable, I volume. Ce fixateur provoque une rétraction qui est inférieure à celle produite par le Bouin (voir résultats tableau $\mathrm{I}$ ).

Après la fixation, des segments d'une longueur d'un centimètre sont prélevés aux trois niveaux précédemment cités. La déshydratation est faite par l'alcool butylique puis les segments sont inclus dans la paraffine. Cette déshydratation entraîne une nouvelle rétraction du tissu qui est estimée par la mesure du segment intestinal lors de la taille du bloc de paraffine.

Des coupes histologiques, d'une épaisseur de 7,5 microns, les unes parallèles à l'axe de l'intestin et passant au niveau de l'insertion du mésentère, les autres perpendiculaires à l'axe, sont effectuées puis colorées par la technique à l'hémalun-éosine.

A l'aide d'un microscope équipé d'un système de projection, les contours de la muqueuse (M) et de la séreuse (S) correspondants sont dessinés, selon les deux orientations des coupes. Ces contours sont mesurés à l'aide d'un curvimètre. Pour les coupes longitudinales, on prend la moyenne des mesures effectuées au niveau de l'insertion du mésentère et du côté diamétralement opposé car le développement de la muqueuse n'est pas le même à ces deux niveaux.

La hauteur des villosités est mesurée sur les projections des coupes longitudinales et ne porte que sur des villosités coupées suivant leur axe, ne présentant qu'une seule assise de cellules jusqu'à leur sommet. Les valeurs sont exprimées en microns.

Le nombre de villosités par centimètre carré de surface séreuse est déterminé sur des photographies de muqueuse fixée, découpée et aplatie, dont la surface correspondante de la séreuse est supérieure au centimètre carré. Le nombre de villosités par centimètre de longueur d'intestin correspond au produit du nombre de villosités par centimètre carré par la longueur de la circonférence intestinale au niveau du segment intestinal étudié. Le prélèvement est au voisinage immédiat du lieu où sont faites les coupes histologiques.

\section{RÉ,SULTATS}

A la lecture du tableau I, il apparaît que : - les rats axéniques et les rats holoxéniques ont un poids semblable, - la présence d'une flore intestinale s'accompagne d'une diminution importante du cæcum mais ne modifie pas la longueur de l'intestin

TABLEAU I

Poids vifs et longueurs d'intestin grêle des deux lots de rats

\begin{tabular}{|c|c|c|c|c|c|c|}
\hline Types de rats & $n$ & Poids (g) & Cæcum (g) & $\begin{array}{c}\text { Longueur } \\
\text { d'intestin grêle } \\
(\mathrm{cm})\end{array}$ & $\begin{array}{l}\text { Longueur } \\
\text { après fixation } \\
(\mathrm{cm})\end{array}$ & $\left\{\begin{array}{l}\text { Rétraction }= \\
\frac{\mathrm{L}_{i}-\mathrm{L}_{f}}{\mathrm{~L}_{i}} \times 100\end{array}\right.$ \\
\hline Holoxéniques & 5 & $212 \pm 3,2$ & $5 \pm 0,13$ & $95,2 \pm 0,29$ & $91,6 \pm 0,4$ & $2,65 \pm 0,38$ \\
\hline Axéniques & 5 & $205 \pm 3,85$ & $26 \pm 0,45(\mathrm{~S})$ & $94,5 \pm 1,16$ & $91,5 \pm 1$ & $3,1 \pm 0,67$ \\
\hline
\end{tabular}

$n$ : nombre d'observations.

Moyenne \pm écart type de la moyenne.

$\mathrm{S}$ : différence significative. 

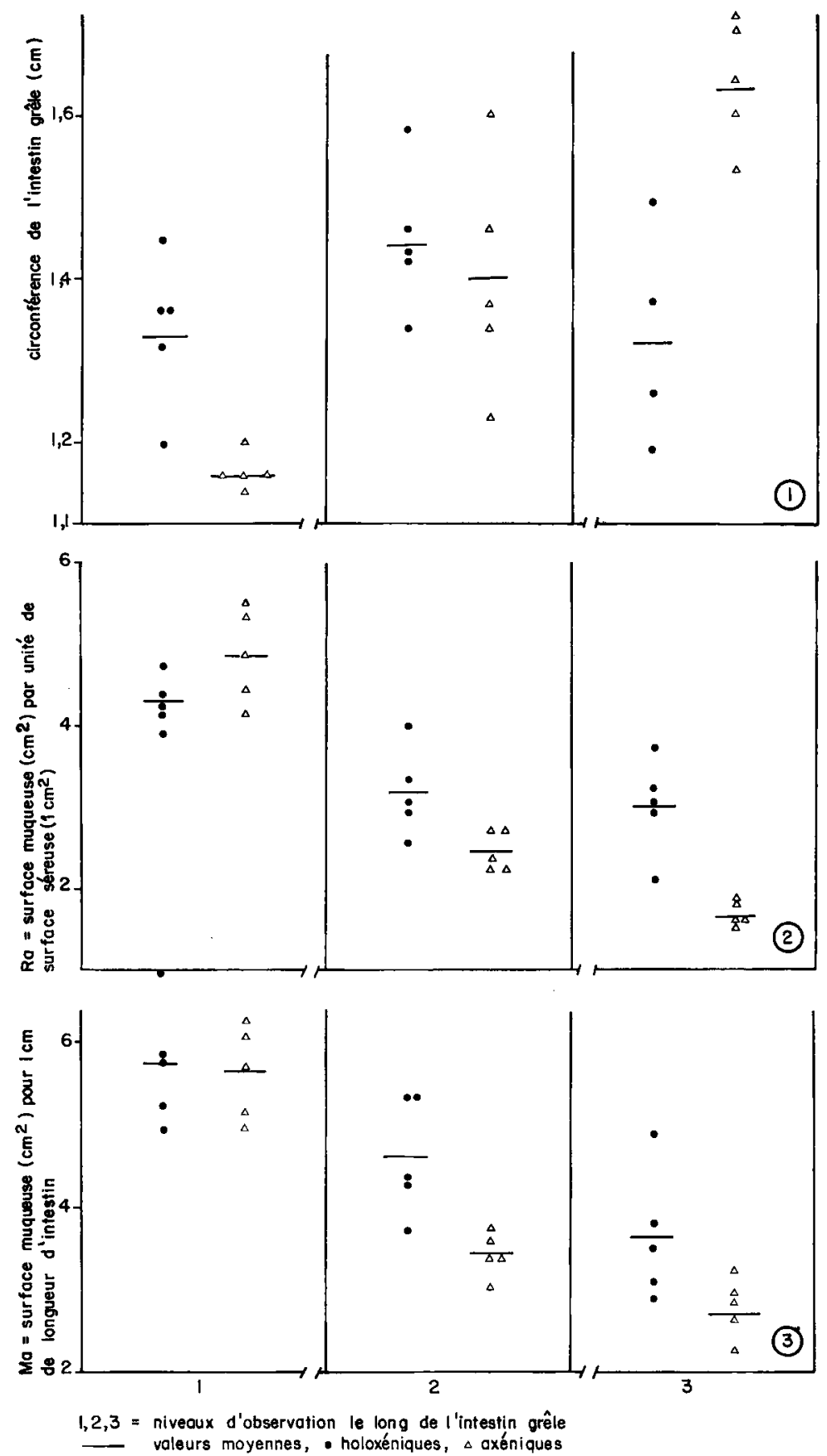

FIG. I-2-3. - I : Circonférence de l'intestin grêle;

2 : Surface muqueuse par unité de surface séreuse;

3: Surface muqueuse par centimètre de longueur d'intestin; aux trois niveaux d'observation. 
grêle - la rétraction au cours de la fixation est de l'ordre de 3 p. Ioo. En outre, la rétraction qui se produit de la fixation à l'inclusion a la même valeur chez les rats des deux groupes et est de l'ordre de $7 \mathrm{p}$. roo. Ainsi l'ordre de grandeur de la rétraction totale est de ro p. roo.

La figure I montre que la présence d'une flore microbienne s'accompagne d'une augmentation de la circonférence intestinale au niveau du duodénum (holoxénique : $\mathrm{I}, 33 \pm 0,04 \mathrm{~cm}$; axénique : I,I $6 \pm 0,00 \mathrm{Im}$ ), d'aucune différence au niveau du jejunum (holoxénique : $I, 44 \pm 0,04 \mathrm{~cm}$; axénique : $I, 40 \pm 0,06 \mathrm{~cm}$ ) et d'une diminution au niveau de l'iléon (holoxénique : $\mathrm{I}, 32 \pm 0,06 \mathrm{~cm}$; axénique : $\mathrm{I}, 53 \pm 0,04 \mathrm{~cm})$. Chez les rats holoxéniques, cette circonférence varie peu du duodénum à l'iléon. Au contraire, chez les rats axéniques, elle augmente du duodénum à 1'iléon.

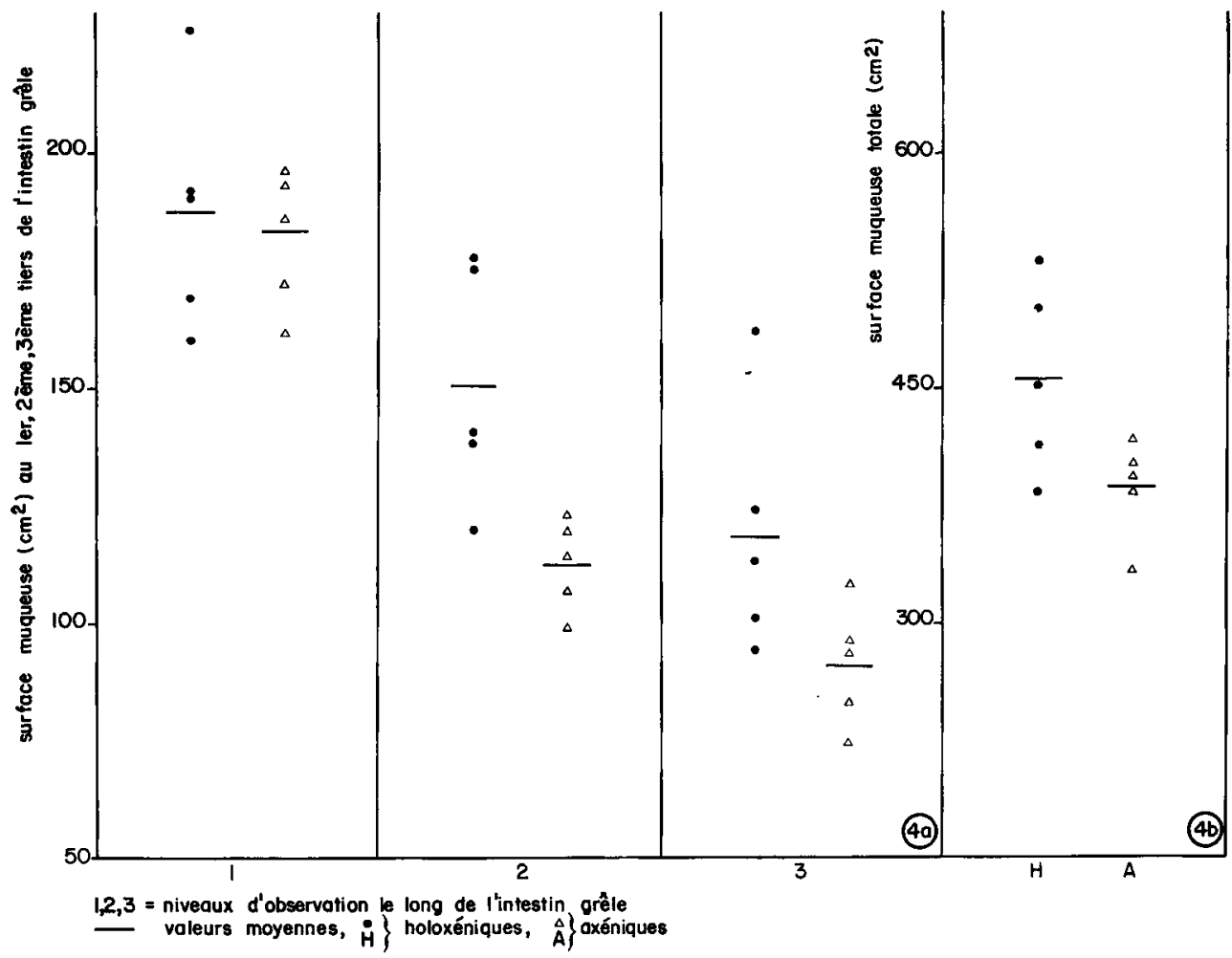

FIG. 4. - 4 a: Surface de la muqueuse à différents niveaux d'observation le long de l'intestin grêle. $4 b$ : Surface muqueuse totale pour le lot holoxénique et le lot axénique.

La surface muqueuse $\mathrm{R} a$, exprimée par rapport à la surface de la séreuse (fig. 2) tend à être plus petite chez les holoxéniques au niveau du duodénum (holoxénique : $4,29 \pm 0,13$; axénique : 4,86 $\pm 0,27$ ), alors que, au contraire, elle est plus grande au niveau du jéjunum (holoxénique : $3,20 \pm 0,25$; axénique : $2,48 \pm 0, \mathrm{II}$ ) et de l'iléon (holoxénique : 3,02 $\pm 0,09:$ axénique : $1,70 \pm 0,07$ ).

Quand la surface muqueuse est exprimée en fonction de la longueur de l'intestin 


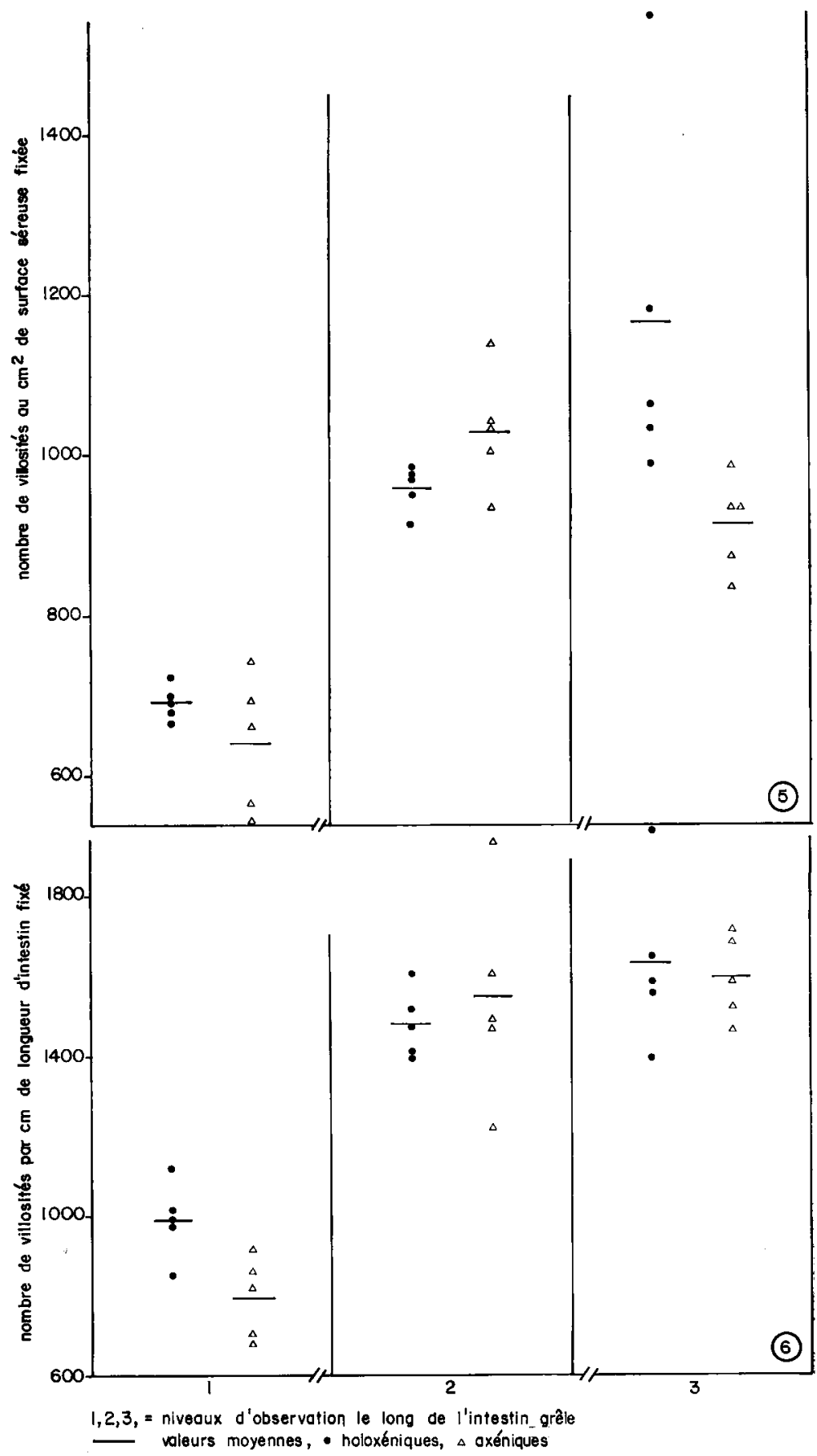

FIG. 5-6. - Nombre de villosités

- 5 au centimètre carré de surface séreuse.

- 6 par centimètre de longueur d'intestin, à différents niveaux d'observation le long de l'intestin. 
grêle (ou $\mathrm{M} a$ ) on n'observe aucune différence au niveau du duodénum (holoxénique : $5,75 \pm 0,35$; axénique : $5,65 \pm 0,38$ ) et les tendances au niveau du jéjunum (holoxé nique : 4,62 $\pm 0,32$; axénique : $3,45 \pm 0,13$ ) et de l'iléon (holoxénique : 3,64 \pm 036 ; axénique : $2,79 \pm 0, \mathrm{I6}$ ) restent les mêmes que celles indiquées pour $\mathrm{R} a$ (voir fig. 3).

L'estimation de la surface réelle de la muqueuse au niveau des 3 segments considérés est représentée par la figure $4 a$. Il n'y a pas de différence de surface de la muqueuse dans le premier tiers de l'intestin grêle (holoxénique : $187,8 \pm \mathrm{I} 2,8 \mathrm{~cm}^{\mathbf{2}}$; axénique : $\left.183,8 \pm 7,5 \mathrm{~cm}^{2}\right)$. Par contre, la surface muqueuse des deux derniers segments intestinaux est plus développée chez les holoxéniques (holoxénique : I50 $\pm 10,8 \mathrm{~cm}^{2}$; axénique : II $2,4 \pm 5, \mathrm{I} \mathrm{cm}{ }^{2}$ au niveau du $2^{\mathrm{e}}$ tiers et holoxénique : II $8,5 \pm$ II $\mathrm{cm}^{2}$; axénique : $9 \mathrm{I}, 5 \pm 5 \mathrm{~cm}^{2}$ au niveau du $3^{\mathrm{e}}$ tiers).

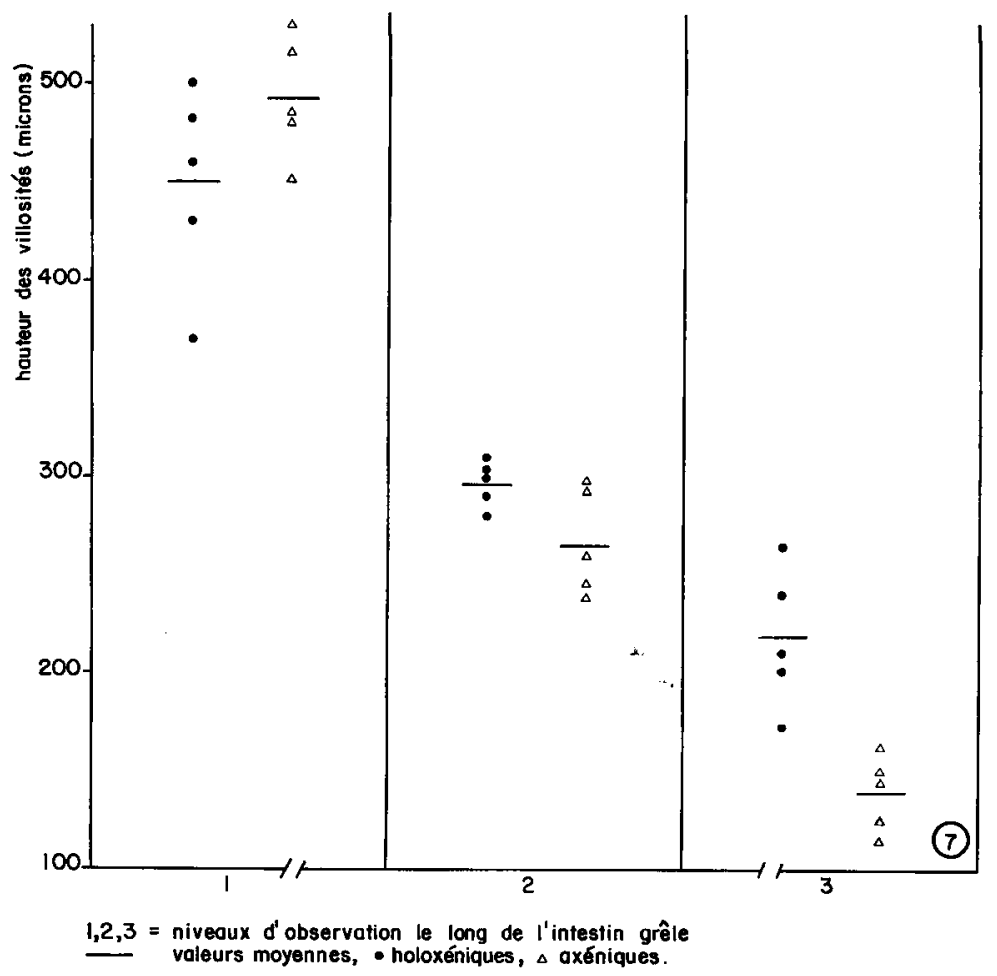

FIG. 7. - Variation de la hauteur des villosités

à différents niveaux de l'intestin grêle chez les rats holoxéniques et les rats axéniques

L'estimation de la surface muqueuse intestinale totale (fig. 4 b), pour du matériel fixé, est de $457 \pm 27 \mathrm{~cm}^{2}$ pour le lot holoxénique et de $390 \pm 14 \mathrm{~cm}^{2}$ pour le lot axénique. Les surfaces muqueuses intestinales correspondant à 1'état frais représentent $500 \pm 30 \mathrm{~cm}^{2}$ pour le lot holoxénique et $429 \pm 16 \mathrm{~cm}^{2}$ pour le lot axénique. L'augmentation de surface muqueuse intestinale totale, due à la présence de la microfore représente I4,2 p. Ioo dans nos conditions expérimentales. Quel que soit le mode d'expression adopté pour cette surface, l'étude statistique montre, compte tenu des variations individuelles et du petit nombre d'animaux étudiés, que cette différence de $I 4,2$ p. IOo n'est pas significative entre les deux lots d'animaux. 
Lorsque l'on rapporte la surface totale au poids corporel la différence entre lots holoxénique et axénique est très réduite (holoxénique : 2,36 ; axénique : 2, Io). I1 n'y a plus aucune différence entre les deux catégories de rats si 1'on exprime la surface muqueuse intestinale totale par rapport au poids corporel déduction faite du poids du contenu cæcal (holoxénique : 2,42 ; axénique $: 2,38$ ).

\section{TABLEAU 2}

Rapport $\frac{\text { surface muqueuse intestinale }\left(\mathrm{cm}^{2}\right)}{\text { poids corporel }(\mathrm{g})}$ d'après divers auteurs

\begin{tabular}{|c|c|c|c|c|c|c|}
\hline & Wood (1944) & $\begin{array}{l}\text { FISHER et al. } \\
\quad(1950)\end{array}$ & $\begin{array}{c}\text { GoRDON et al. } \\
(1961)\end{array}$ & $\begin{array}{c}\text { HROMADKOVA } \\
\text { et al. (1968) }\end{array}$ & \multicolumn{2}{|c|}{$\begin{array}{c}\text { MesLin (1972) } \\
\text { SACQUet, Guenet }\end{array}$} \\
\hline $\begin{array}{l}\text { Rats } \\
\text { holoxéniques }\end{array}$ & $\frac{680}{295}=2,30$ & $\frac{700}{293}=2,39$ & $\frac{671}{320^{*}}=2,10$ & $\frac{436}{210}=2,08$ & $\frac{500}{212}=2,36$ & $\frac{500}{207^{*}}=2,42$ \\
\hline $\begin{array}{c}\text { Rats } \\
\text { axéniques }\end{array}$ & & & $\frac{468}{312^{*}}=1,49$ & & $\frac{429}{205}=2,10$ & $\frac{429}{180^{*}}=2,38$ \\
\hline
\end{tabular}

* Poids vif, déduction faite du poids du contenu cæcal.

Le nombre de villosités au $\mathrm{cm}^{2}$ de surface séreuse d'une part et le nombre de villosités par cm de longueur d'intestin d'autre part sont représentés respectivement par les figures 5 et 6 . La première montre une très grande dispersion de certains résultats. Cependant, au niveau de l'iléon, malgré la diminution relative de la surface séreuse chez les rats holoxéniques, le nombre de villosités par $\mathrm{cm}^{2}$ de surface séreuse apparaît plus élevé chez ces sujets.

La seconde figure représente le nombre de villosités par centimètre de longueur d'intestin. Il y a une très nette augmentation de ce nombre dans les deux groupes de sujets du duodénum vers l'iléon. Ce nombre est significativement plus élevé chez les rats holoxéniques au niveau du duodénum (holoxénique : $992 \pm 4 \mathrm{I}$; axénique : $797 \pm 46$ ). Les différences de variance au niveau du jéjunum sont anormalement importantes et ne permettent pas de faire une comparaison valable. Aucune différence ne semble exister au niveau de l'iléon entre les deux groupes de sujets (holoxénique : I $632 \pm 96$; axénique : I $595 \pm 48$ ).

La hauteur des villosités mesurée sur 20 villosités pour chaque niveau et chaque animal, décroît du duodénum à l'iléon dans les deux groupes d'animaux (fig. 7). Chez les holoxéniques, cette hauteur tend à être plus faible au niveau du duodénum (holoxénique : $45^{\circ} \pm 22$ microns; axénique $495 \pm$ I6 microns) mais la différence ne peut être affirmée; au contraire, cette hauteur est plus importante au milieu du jéjunum (holoxénique : $300 \pm 7$ microns ; axénique : $220 \pm$ I 8 microns) et encore plus au niveau de l'iléon (holoxénique : $220 \pm$ I 8 microns ; axénique : $140 \pm 9$ microns). 


\section{DISCUSSION}

\section{A. - Rétraction}

Nous n'avons pas mesuré la rétraction de la circonférence intestinale, vu les difficultés de mesure à l'état frais. Pour SOERGEL (I969) rien ne prodve que la rétraction affecte au même degré et dans toutes les directions les différentes tuniques de 1a paroi intestinale si celle-ci n'est pas mesurée selon les deux orientations. C'est pour cette raison que nous avons utilisé un fixateur et un agent de déshydratation qui produisent une rétraction minimale par rapport à l'état frais. Toutefois nous n'avons pas observé sur les coupes histologiques de décollement des différentes tuniques intestinales les unes par rapport aux autres et ceci peut conduire à penser, après Fisher et PARsons (I950) que la rétraction se fait de manière égale dans toutes les directions.

\section{B. - Longueur intestinale}

Hromadkova et Skala (I968) indiquent une longueur d'intestin grêle à l'état frais de 96,9 $\pm 5,6 \mathrm{~cm}$ chez des rats Wistar de 170 à 2 ro $\mathrm{g}$. Les données que nous obtenons chez les rats holoxéniques $F$ ischer confirment celles de ces auteurs. Pour Fischer et Parsons (I950), chez le rat Wistar, la longueur de l'intestin grêle est indépendante du poids de l'animal au-delà de $15^{\circ} \mathrm{g}$ et a une valeur moyenne de III $\mathrm{cm}$. Pour des rats de même lignée, de poids compris entre I60 et 2Io g elle est de $84,9 \pm 5,9 \mathrm{~cm}$ (GoRDON et WostmanN, I960). Les différences entre ces résultats peuvent être liées à la lignée, au régime alimentaire et à la méthode de mesure de la longueur intestinale.

Comme GoRdon et al., (I960-Ig66) nous n'observons pas de différence significative de la longueur de l'intestin grêle entre rats holoxéniques et rats axéniques.

\section{C. - Circonférence intestinale}

Pour GoRdon et BRUCKNER-KARdoss (I96r), des rats holoxéniques et axéniques de même sexe, âge, poiđs, recevant le même régime alimentaire présentent une circonférence intestinale comparable. Ces auteurs cependant rapportent que des difficultés techniques les ont empêchés de maintenir constante la pression hydrostatique dans l'intestin au cours de la fixation et mentionnent que la pression intraluminaire apparaissait faible à l'observation visuelle.

Les longueurs de circonférence intestinale que nous indiquons correspondent aux valeurs mesurées sur les coupes histologiques. Nous obtenons pour des intestins fixés successivement sous pression constante des résultats reproductibles et des valeurs bien groupées. La longueur de la circonférence intestinale au niveau du duodénum et de l'iléon est différente chęz les rats axéniques et chez les rats holoxéniques Ceci ne s'accorde pas avec les résultats obtenus par les auteurs précédemment cités.

Il est possible que l'augmentation de circonférence intestinale au niveau de l'iléon chez le rat axénique provienne d'une diminution du tonus de la musculeuse 
à ce niveau, en relation avec la diminution du tonus musculaire observée au niveau du cæcum chez certains rongeurs dans l'état de vie axénique (FALK, I960 ; GoRDON, I968).

Les autres données fournies par la littérature chez les rats holoxéniques concernant la circonférence intestinale sont aussi variables que celles qui se rapportent à la longueur de l'intestin grêle : $1,93 \mathrm{~cm}$ pour du matériel fixé au Bouin et $\mathrm{I}, 70 \mathrm{~cm}$ pour du matériel inclus dans la paraffine (Fisher et PARSONS, 1950); $1,06 \mathrm{~cm}$ à l'état frais et $\mathrm{I}, 38 \mathrm{~cm}$ au milieu du jéjunum après fixation in situ (Hromadkova et SkAL,A. I968; $0,87 \mathrm{~cm}$ au milieu du jéjunum pour du matériel fixé au Bouin GORDON et BRUCKNER-KARDOSS, I96I).

\section{D. - Surface muqueuse intestinale}

Selon Fisher et Parsons (I950) la surface muqueuse par centimètre de longueur d'intestin grêle, $\mathrm{M} a$, décroît linéairement du pylore à la jonction iléo-cæcale chez le rat Wistar. Les valeurs que nous obtenons chez les rats Fischer holoxéniques, bien qu'elles soient très variables donnent des moyennes qui effectivement s'alignent.

Chez les sujets axéniques, il en est autrement. Les valeurs observées sont beaucoup mieux groupées que chez les rats holoxéniques mais les moyennes ne s'alignent pas. Il est donc douteux que chez les axéniques la surface décroisse linéairement en fonction de la longueur. En conséquence, l'estimation de la surface de la muqueuse intestinale chez ces sujets devient difficile et ne peut être qu'approximative.

Nous avons rassemblé dans le tableau 2 les valeurs du rapport surface muqueuse intestinale/poids corporel observées par divers auteurs et par nous-mêmes.

Des différences importantes apparaissent d'un auteur à l'autre : les valeurs extrêmes diffèrent de I3 p. Ioo chez les rats holoxéniques. Les résultats que nous obtenons chez ce genre de sujets s'accordent avec ceux de Woon ainsi que ceux de Fisher et Parsons, alors que les résultats de Gordon et Bruckner-Kardoss se rapprochent de ceux de Hromadkowa et al., mais surtout, comme nous l'avons déjà fait remarquer, les rats axéniques, si l'on tient compte du poids anormal de leur cæcum, ne diffèrent pas, d'après nos estimations, des rats holoxéniques alors que, d'après GORDON et BRUCKNER-KARDOSS, le rapport surface muqueuse intestinale/poids corporel observé chez les sujets axéniques est beaucoup plus faible que chez les sujets holoxéniques : la différence atteint $30 \mathrm{p}$. Ioo par rapport à la valeur observée chez les holoxéniques et $40 \mathrm{p}$. Ioo par rapport à la valeur observée chez les axéniques.

Il est possible que ces écarts entre les résultats obtenus par ces derniers auteurs et ceux que nous obtenons résultent des différences importantes entre les techniques utilisées et de l'imprécision de la méthode de mesure chez le rat axénique. Ils peuvent aussi provenir de la lignée des rats, de la nature de l'aliment et des conditions de milieu : les sujets axéniques étudiés par GoRdON et BRUCKNER-KaRDOsS vivaient dans un état de surpopulation tel qu'ils se trouvaient soumis à un stress de longue durée (Gordon et Wostmann, I96o).

\section{E. - Hauteur des villosités}

Chez les rats holoxéniques, la hauteur moyenne des villosités est semblable à celle que rapportent Hromadkova et SkaLA (I968) (264 \pm 42 microns au milieu du jéjunum $225 \pm 42$ microns à la fin de l'iléon) et, compte tenu du poids des sujets, 
est du même ordre de grandeur que celle rapportée par Altmann et EnEsco. (1967).

Nous ne pouvons comparer la hauteur moyenne des villosités à différents niveaux de l'intestin grêle chez les rats axéniques puisque GORDON et BRUCKNER-KARDOSS (I96I) indiquent qu'ils n'en ont pas effectué la mesure. Cependant, dans un travail récent sur le duodénum de rat, GALJAARD et al. (I972) décrivent des villosités moins grandes chez le sujet holoxénique par rapport à l'animal axénique ; nos résultats, pour ce niveau d'observation, vont dans ce sens. Chez la souris, au niveau de l'iléon, le nombre de cellules épithéliales de la base au sommet de la villosité est significativement plus grand chez l'animal holoxénique, de l'ordre de ro p. Ioo (ABRAms et al., I963). Nous observons une différence dans le même sens concernant la hauteur des villosités chez le rat, mais elle est plus importante.

Comment la flore microbienne intestinale modifie-t-elle ce caractère? Selon AltmanN et LEBLOND (I967-I970), chez le rat holoxénique le gradient de taille des villosités dépend de la composition du contenu intestinal, composition qui est très différente du duodénum à l'iléon. En particulier, la sécrétion pancréatique provoquerait un allongement de la longueur des villosités au niveau du duodénum (ALTMANN, I97I), alors qu'au contraire il existerait dans le chyme iléal des substances qui réduiraient cette longueur. Chez le rat axénique ces deux actions seraient encore plus accusées que chez le rat holoxénique; la flore microbienne tendrait à détruire, chez le rat holoxénique, les substances actives. Cette action destructrice serait peu importante au niveau du duodénum, lieu où la flore microbienne est peu abondante (SAcqueT, RAIBAUd et GaRnIER, I97 $x$ ) et où le transit gastro-intestinal est très rapide (MARCuS et LENGEMANN, I962). Elle serait au contraire plus marquée au niveau de l'iléon où la flore microbienne est plus abondante et le transit plus lent.

Reçu pour publication en novembre 1972.

\title{
SUMMARY
}

\author{
ACTION OF BACTERIAL, FLORA \\ ON THE MORPHOLOGY AND MUCOUS SURFACE \\ OF THE SMALI, INTESTINE OF THE RAT
}

A comparative study of the morphology of the small intestine in axenic and holoxenic rats brings to light large morphological differences which express the action of the microbial flora on that organ.

Various morphological characters, such as length of small intestine circumference, mucous surface area, number and height of villi, are not modified in a uniform way at all levels. In axenic rats, length of circumference decreases in the anterior region, remains unchanged in the middle region and increases in the posterior region, while the mucous surface area is similar in the anterior region and decreases in the middle and terminal regions.

The number of villi is decreased in the anterior region and is unchanged in the other regions, while villosity height tends to be greater in the anterior region and less in the other regions.

Total intestinal mucous surface area, expressed in terms of rat body weight, is not significantly less in the axeric subject. 


\section{RÉFÉRENCES BIBLIOGRAPHIQUES}

Abrams G. D., Bauer H., Sprinz H., 1963. Influence of the normal flora on mucosal morphology and cellular renewal in the ileum. A comparison of germ-free and conventional mice. Lab. Invest., 12, $355-364$.

Altmann G. G., Enesco M., I967. Cell number as a measure of distribution and renewal of epithelal cells in the small intestine of growing and adult rats. Am. J. Anat., 121, 319-336.

Altmann G. G., Leblond C. P., r 967 . A duodenal factor in the control of villus size in the small intestine of the rat (abstract). Anat. Rec., 157, 205.

Altmann G. G., Leblond C. P.y I97o. Factors influencing villus size in the small intestine of adult rats as revealed by transposition of intestinal segments. Am. J.Anat., 127, 15-36.

Altmann G. G., I97I. Influence of bile and pancreatic secretions on the size of the intestinal villi in the rat. Am. J. Anat., 182, 167-178.

Boyne R., Fell B. F, RobB I., 1966. The surface area of the intestinal mucosa in the lactating rat J. Physiol., 188, 570-575.

FALK J. L., I960. Editorial : cæcal enlargement in germfree animals. Nutr. Rev., 18, 3I3-3r4.

Fisher R. B., Parsons D. S., r950. The gradient of mucosal surface area in the small intestine of the rat. J. Anat., 84, 272-282.

GaljaArd H., van der Meer-Fieggen W., Giesen J., i972. Feedback control by functional villus cells on cell proliferation and maturation in intestinal epithelium. Exp. Cell. Res., 73, I97-207.

Gordon H. A., 1960. The germ-free animal. Its use in the study of " physiologic " effects of the normal microbial flora on the animal host. Am. J. Digest. Diseases, New Series, 5, $84 \mathrm{I}-867$.

Gordon H. A., Wostmann B. S., 1960. Morphological studies on the germ-free albino rat. Anat.

1. Rec., 137, 65-70.

Gordon H. A., Bruckner-Kardoss E., 1961. Effect of normal microbial flora on intestinal surface area. Amer. J. Physiol., 201, r75-I78.

Gordon H. A., Bruckner-Kardoss E., I96r. Effects of the normal microbial flora on various tissue elements of the small intestine. Acta Anat., 44, 210-225.

Gordon H. A., Bruckner-Kardoss E., Staley T. E., Wagner M., Wostmann B, S., 1966. Characteristics of the germ-free rat. Acta Anat., 84, 367-369.

Gordon H. A., I968. Is the germ-free animal normal? A review of its anomalies in young and old age. In : "The germ-free animal in research" chap. $6: 127-150$, M. E. Coates Éd. Academic Press, I968.

Hromadkova V., Skala I., rg68. Factors influencing the assessment of size of the mucosal surface and length of the small intestine in rats. Digestion, 1, I49-158.

Marcus C. S., Lengemann F. W., I962. Use of radio-yttrium to study food movement in the small intestine of the rat. $J . N u t r ., 78$, I79-I82.

Permezel N. C., Webling D. D'A., 197I. The length and mucosal surface area of the small and large gut in young rats. J. A nat., 108, 295-296.

Raibaud P., Dickinson A. B., Sacouet E., Charlier H., Mocouot G., ig66. La microflore du tube digestif du rat. IV. Implantation contrôlée chez le rat gnotobiotique de différents genres microbiens isolés du rat conventionnel. Ann. Inst. Pasteur, 111, 193-21o.

Sacquet E., Raibaud P., Garnier J., ig7i. Étude comparée de la microflore de l'estomac, de l'intestin grêle et du cæcum du rat " holoxénique " (conventionnel), et de ses modifications à la suite de diverses interventions chirurgicales : anse aveugle jéjunale, déviations biliaires. Ann. Inst. Pasteur. 120, $50 I-524$.

Soergel K. H., Ig69. Measurement of intestinal absorptive surface area. Gastroenterology, 56, 808-809.

WARREN R., 1939. Serosal and mucosal dimensions at different levels of the dog's small intestine. Anat. Rec., 75, 427-437.

WooD H. O., r944. The surface area of the intestinal mucosa in the rat and in the cat. J. Anat., 78, 103-105. 